\title{
EDITORIAL
}

\section{Here is the new name and face}

Here we are with the first issue of the International Journal of Clinical Pharmacy. The journal that was called Pharmacy World \& Science has changed its name. But all editors, editorial staff and reviewers will remain concentrated on this journal under the new title.

The focus of the journal will remain the same too. We still welcome articles on clinical and social pharmacy, pharmacotherapy and pharmaceutical care. Will all remain the same then? That remains to be seen.

The world around us is continuously changing, and so is the profession of pharmacy. We have known the ages of therapeutic drug monitoring, of drug-drug interactions, of patients counselling and standards and protocols. We've lived through the rise of evidence based medicine and pharmacy. Newer topics come up continuously like medication errors, pharmacogenomics and reviewing medication. Different countries run at different pace and yet, pharmacists are still there to serve society, to serve 'our patients'. Knowledge is developing continuously, and that als means change in the professional activities of (clinical) pharmacists.
The profession of pharmacy is a real profession, and therefore pharmacists should be able to look at each patient as if it was the first. There are many evidence based treatment guidelines. But there can be many reasons why such guidelines do not fully apply. Such reasons are usually related to characteristics of the individual patient. Be it social circumstances, multimorbidity, a certain genetic make-up, nutritional status, or simply allergies and drug-drug or drug-food interactions. This issue of the International Journal of Clinical Pharmacy is largely dedicated to individualised pharmacotherapy. For this first issue of the journal under its new name, a number of experts have prepared reviews or research reports that relate to this topic especially for this issue.

We wish you a good reading of the International Journal of Clinical Pharmacy, now and in the future.

Dr. J.W. Foppe van Mil, Editor-in-chief

Dr. Marie Caroline Husson, Member of the editorial board

Prof. Dr. Peter A.G.M. De Smet, Member of the editorial board 